\title{
Kernos
}

Revue internationale et pluridisciplinaire de religion grecque antique

9 | 1996

Varia

\section{Augusto FRASCHETTI, Rome et le prince}

\section{Bruno Rochette}

\section{(2) OpenEdition \\ Journals}

\section{Édition électronique}

URL : http://journals.openedition.org/kernos/1197

DOI : 10.4000/kernos. 1197

ISSN : 2034-7871

Éditeur

Centre international d'étude de la religion grecque antique

Édition imprimée

Date de publication : 1 janvier 1996

ISSN : 0776-3824

\section{Référence électronique}

Bruno Rochette, «Augusto fraschett, Rome et le prince », Kernos [En ligne], 9| 1996, mis en ligne le 16 juin 2011, consulté le 24 septembre 2020. URL : http://journals.openedition.org/kernos/1197 ; DOI : https://doi.org/10.4000/kernos.1197 
de même que des banquets (?). Le parèdre de la Grande Déesse de l'île, dont l'activité métallurgique conviendrait bien à un Volcanus indigène, reproduirait le "schéma" du Velchanos crétois, peu à peu identifié à Apollon. Héphaïstos est ensuite appelé à la barre, en ses cultes lemnien et athénien, où l'A. retrouve, une fois encore, avec des déformations qui seraient dues à autant de réinterprétations, les données mises au jour dans les îles égéennes.

La troisième et dernière partie nous ramène en Étrurie, à la recherche d'une définition du dieu*Velchans, en un long développement dont je ne suis pas à même d'évaluer la pertinence, mais qui fait appel à la fois aux légendes et aux cultes locaux. Et c'est avec la conclusion que l'A. reprend l'ensemble des acquis pour redéfinir la personnalité du Volcanus latin.

Ce travail, dont la présentation est irréprochable, est une véritable thèse qui met à son service un comparatisme fouillé et une remarquable documentation. Une foule de questions, dont l'importance et l'intérêt sont variables, sont aussi abordées dans les notes. La plupart des textes anciens discutés - dont l'importance pour le propos est également très variable - sont reproduits intégralement dans leur version originale avec, systématiquement, une traduction de l'A. en note. Une telle recherche est très séduisante et satisfait l'aspiration à comprendre des traditions éclatées dans une mise en perspective globalisante. L'A. propose maints rapprochements dont la convergence emporte souvent l'adhésion. Néanmoins, malgré la prudence dont il fait preuve en rappelant fréquemment le caractère hypothétique de bon nombre d'interprétations, on est pris de vertige devant une recontruction dont les assimilations comparatistes ne permettent pas de justifier toutes les étapes.

Vinciane PIRENNE-DELFORGE (Université de Liège)

Augusto Fraschetri, Rome et le prince. Traduction française par Vincent Jolivet, Paris, Belin, 1994. 1 vol. 14 x 20,5 cm, 380 p. (L'Antiquité au présent). ISBN : 2-7011-1478-0.

Nicole Loraux et Yan Thomas ont eu l'excellente idée d'accueillir dans leur collection, L'Antiquité au présent, une version française de l'ouvrage d'Augusto Fraschetti, Roma e il principe (Roma-Bari, 1990). Le sujet n'est pas neuf : étudier de quelle manière et dans quelle mesure la vie du citoyen romain a été modifiée, spécialement dans le domaine religieux, par les changements dus au passage de la République au Principat, étape cruciale de l'histoire romaine que R. Syme a naguère qualifiée à juste titre de Révolution romaine. C'est le problème le plus épineux posé par le Principat, qui a fait couler des flots d'encre, qu'affronte l'A. : le rapport du Princeps et de l'Urbs et la manière dont s'insèrent dans les structures existantes de l'État romain éléments politiques et religieux pour former l'auctoritas Augusta. La nouveauté propre à cette étude, c'est l'angle selon lequel la matière est abordée. L'A. n'hésite pas à remettre en débat des questions qui passent généralement pour résolues depuis longtemps - en fait depuis Mommsen - et à faire un sort à des problèmes généralement considérés comme de simples curiosités d'érudits ou d'antiquaires. Fondée une quantité de textes minutieusement étudiés, sa réflexion 
s'opère en trois étapes : le temps, l'espace et les cultes de la cité et de la maison du Prince.

L'instauration d'un État dirigé par un seul homme a engendré des modifications chronologiques dans la vie de la cité. L'introduction de nouvelles fêtes religieuses et de nouveaux jours fériés célébrant l'anniversaire d'une victoire ou d'un événement heureux quelconque entraîne une nouvelle manière de vivre et de comprendre le rythme du temps dans le quotidien du ciuis Romanus. Le calendrier réformé de César - manipulé à des fins politiques - accueillait déjà des feriae publicae commémorant les grandes victoires du dictateur à leur jour anniversaire. L'année augustéenne est elle aussi scandée par le «temps du prince », qui devient, par une manipulation et, pour ainsi dire, une conquête du temps civique, le "temps de la cité ». Les ciues vivent au rythme dicté par leur dirigeant. Aux jours d'allégresse se joignent les temps du deuil, qui se traduisent en cérémonies spécifiques. Les funérailles de César donnèrent lieu à une laudatio, comme déjà celles de Sylla. La mort d'Auguste fut suivie d'un deuil public. Fort habilement, le Princeps a su ainsi créer une stratégie du deuil, au caractère politique évident, en impliquant toute la cité dans les décès qui frappaient la domus Augusta. Tibère agira encore de même le jour où il perdit Germanicus, même s'il fit en sorte de limiter les conséquences provoquées par les malheurs familiaux dans la cité.

L'autre dimension sur laquelle joue Auguste, c'est l'espace, le cadre territorial de la cité, qu'il redéfinit dans un but précis. Les subdivisions du spatium Urbis - le tissu urbain de Rome -, qui remontent aux origines de la Ville, ont une valeur symbolique. Les mutations qui l'affectent ne peuvent s'expliquer uniquement par des nécessités fonctionnelles. C'est d'une idéologie que procède la réorganisation de l'espace de l'Urbs. Auguste introduit un nouveau système de partition, celui des $u i c i$, qui rendait obsolètes les anciennes formes de subdivision, les montes et les pagi. Cette nouvelle organisation a des conséquences sur certaines fêtes religieuses, comme les Compitalia, jours de bombances et de jeux - restaurés par Auguste - qui rassemblaient dans les carrefours les Lares des différents domaines et les familiae. Après s'être penché sur la subdivision du spatium Urbis à la fin de la République (montes et pagi) et sur les problème des $u i c i$ à l'époque de Cicéron et de Clodius, où ils apparaissent comme des lieux de subversion, l'A. étudie avec minutie les témoignages de Suétone et de Dion Cassius qui présentent le système de partition structurée en uici du spatium Urbis comme une innovation d'Auguste.

Dans une cité ainsi redéfinie dans le temps et dans l'espace, Auguste n'éprouvera aucune difficulté à assimiler les cultes de la cité à ceux de sa domus, puisque, désormais, les Pénates du peuple romain sont confondus avec ceux d'Auguste en

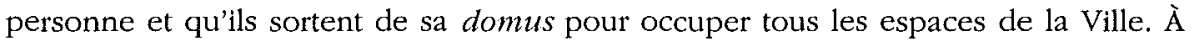
partir de la mort d'Agrippa, en 12 av. J.-C., qui eut droit à sa laudatio, les morts remarquables de la domus Augusta seront mis au service de l'idéologie du Prince. Mais il y a plus. Loin de se contenter de simples assimilations, Auguste va jusqu'à se découvrir de véritables liens de parenté avec certaines divinités. Ainsi avec Vesta. Dans les Fastes (III, 417-426), Ovide parle, à propos de cette déesse et d'Auguste, de cognata numina (425-6) et de cognatum caput (426). La cognatio d'Ovide renverrait à l'étroit lien unissant la déesse et le Prince, à moins qu'il ne s'agisse d'une simple allusion à l'idéologie officielle du régime, faisant d'Auguste le protecteur de Rome au 
même titre que Vesta, qui a les mêmes origines troyennes que le Prince ${ }^{3}$. Quoi qu'il en soit, que Vesta ait revêtu une grande importance aux yeux d'Auguste, sa mention dans les Res gestae $(21,2)$ le prouve. De plus, c'est à cette déesse qu'Auguste fit élever, en 12 av. J.-C., une statue sur le Palatin et ériger un autel au même endroit, sans aller toutefois jusqu'à lui faire construire un temple, comme on l'a maintes fois soutenu, qui eût été la réplique de celui du forum où était conservé le feu sacré.

Les idées nouvelles contenues dans ce livre n'emporteront certes pas l'adhésion de tous. Elles ne laisseront en tout cas personne indifférent. Parmi les points de vue qui me paraissent un peu trop hardis, il faut signaler l'idée qu'Auguste institua un rapport nouveau entre hommes et dieux. Cette manière de concevoir la religion est loin d'être une innovation propre au début du Principat. On la trouve, sous une forme un peu différente, il est vrai, dès la deuxième guerre punique, lorsque $P$. Cornélius Scipion l'Africain, proclamé imperator sur le champ même de bataille sans être magistrat -, reçut la protection de l'auctoritas de Jupiter Capitolin, qui lui accorde l'imperium. Et ce n'est pas un cas unique: Sylla est dit felix, favorisé par les dieux, Pompée, dit Magnus, est le protégé de Jupiter Maximus, et César, imperator et parens patriae, est assimilé à la vieille divinité Romulus-Quirinus, lien qui annonce son apothéose, réplique de celle de Romulus.

Ce travail, qui repose sur une connaissance approfondie des sources anciennes et de la bibliographie moderne, constitue une contribution de valeur à l'étude d'un phénomène complexe.

Bruno RocheTTE

(Université de Liège)

María Cruz Herrero Ingelmo, Pausanias. Descripción de Grecia, Introducción, traducción y notas, Madrid, Editorial Gredos, 1994, 12,5 x $19,5 \mathrm{~cm}$, vol. I, libros I-II, 358 p. +4 cartes; vol. II, libros III-VI, 435 p. +3 cartes; vol. III, libros VII-X, 529 p. + 4 cartes (Biblioteca Clásica Gredos, 196, 197, 198). ISBN : 84-249-1650-6.

La science de la Philologie Classique se développe au rhytme de la parution de nouvelles éditions, études, lexiques, dictionnaires, mais on ne doit pas en oublier pour autant la valeur des traductions, fondamentales en tant que courroie de transmission pour une société ignorant de plus en plus les langues et les cultures antiques.

En Espagne, des groupes très importants de spécialistes de la civilisation antique se sont développés, qui prétendent situer la culture classique dans son époque de splendeur, le Siècle d'Or espagnol. Dans cette atmosphère, on doit saluer le progrès fait par la maison d'édition Gredos : sa Biblioteca Clâsica compte déjà les deux cents volumes. Les introductions à chaque volume ont acquis un prestige mérité, constituant une sobre mise à jour des problèmes essentiels de chaque ouvrage. Il est donc naturel de donner à la connaissance de la communauté scientifique internationale cette collection, destinée fondamentalement à un public cultivé de langue espagnole, mais qui a aussi une certaine valeur pour les spécialistes en général. 